\title{
EVALUATION OF KIDNEY STATUS BY ULTRASONOGRAPHY IN CANINES: AN EXPERIMENTAL STUDY
}

\section{Ditul Barman* and Rajiv V. Gaikwad}

Department of Veterinary Medicine, Bombay Veterinary College, Parel

Mumbai-400012, India

*Corresponding author: Ditul Barman, E-mail: ditulbarman@rediffmail.com

\section{ARTICLE INFO}

\section{ABSTRACT}

\begin{abstract}
Received
19.09.2014

Accepted

27.11.2014

Online

27.12.2014

Key words:

Kidney

Ultrasonography

Creatinine

Echogenic

Canine

Kidney is a major organ for homeostasis of the body's extracellular fluids by maintaining a constant internal environment. By playing such crucial responsibility kidney remains vulnerable for various diseases and disease condition. Other than laboratory findings, application of ultrasonography can be considered as one of the finest diagnostic tool to determine the architectural detail of kidney. To compare the study, we have taken two groups of canine, namely healthy and clinical. The later grouped was screened based on clinical signs, Blood Urea Nitrogen (BUN) and Serum Creatinine value. Sonography was performed by using Brightness mode instrument for both the groups. The values were analyzed by Prolate Ellipsoid formula to estimate the volume of kidney. There was significant variation in sizes between the kidneys of both the groups. Moreover, the size of the kidneys of few clinical cases, which has been suffering from renal disorder since long, reveals smaller size kidney than the healthy groups. Thus, this noninvasive method is quite practicable to evaluate the size of kidney amongst various renal failure cases.
\end{abstract}

To cite this article: D Barman and RV Gaikwad, 2014. Evaluation of kidney status by ultrasonography in canines: an experimental study. Res. Agric., Livest. Fish. 1(1): 105-108.

This article is an open access article licensed under the terms of the Creative Commons Attribution License.

www.agroaid-bd.org/ralf, e-mail: editor.ralf@gmail.com 


\section{INTRODUCTION}

Kidney is a vital and complex organ in all mammals. The major function of kidney is urine formation and to excrete nitrogenous waste products, ammonia, urobilin, myoglobin, creatinine, uric acid, aliphatic amines and several other toxic materials. Renal disorders are most exaggerated when $75 \%$ or more of the nephrons turn nonfunctional. Detection of specific renal disorder before aggravating serious impairment of the renal function is quite difficult because of the tremendous reserve capacity and marked functional adaptation, which is significant characteristic of the kidney. Abdominal ultrasonography has been used in veterinary medical research to define normal status of anatomic determinants and so helpsF in recognition and evaluation in kidney diseases. It is advantageous in assessing the architecture of kidneys that do not pacify during urography (Walter et al., 1987). Ultrasonography is an imaging modality in animals since it is a non-invasive and presents no known hazard to the operator as well as patients. The present research work was undertaken to study canine kidney structure of normal and renal disease cases employing ultrasonography.

\section{MATERIALS AND METHODS}

The clinical cases were selected at the Out Patient Department (OPD) and at the Medical Ward of Bombay Veterinary College, Parel, Mumbai - 400012 from both in-patient and out-patient perspective on the basis their clinical sign i.e. gastrointestinal signs, dehydration, stomatitis, polyuria, polydipsia and blood biochemistry of BUN $\geq 20 \mathrm{mg} / \mathrm{dl}$ and Serum Creatine $\geq 2 \mathrm{mg} / \mathrm{dl}$. The apparently healthy groups were selected to standardize the baseline data with age ranging from 29 years. Transabdominal Ultrasonography was performed by means static B-scan or B-mode instrument ${ }^{*}$ using a dual frequency $(3.5 / 5.0 \mathrm{MHz})$ curved array transducer ${ }^{* *}$. For documentation, a video graphic printer*** was used to record the image. The Right Kidney was scanned by placing the probe caudal to the Right costo-spinal area where as the Left Kidney was scanned by placing the probe caudal Left costo -spinal area in dorsal recumbence position of the patient at the level of $2^{\text {nd }}$ to $3^{\text {rd }}$ lumber vertebra.

*Scanner 200VET (Pie Medical Equipment B. V., Philipsweg 1, 6227 A J Maastricht, Netherlands); **80F R40 (Pie medical Equipment B. V., Philipsweg 1, 6227 A J Maastricht, Netherlands); ${ }^{* * *}$ Sony up -890 MD/2 (SYN) Video Graphic printer, Sony Corporation, Tokyo, Japan.

By using B mode (brightness mode) we have taken different images of kidney. It is also called as real time scanning. After freezing the images with the help of the user manual, different parameter were noted. In order to find out the volume of each kidney all recorded parameters were then put in a formula of Prolate Ellipsoid method and calculated. (Felkei et al. 1992). $V=A 2 x$ $0.85 / L$, where $V=$ volume, $A=$ area and $L=$ length of the kidney.

\section{RESULTS AND DISCUSSION}

Left and Right kidney of clinical groups showed significant reduction in volume than healthy group. The mean kidney volume of left and right kidney of healthy group was $(44.53 \pm 3.49 \mathrm{~cm} 2)$ and $(47.39 \pm 4.3 \mathrm{~cm} 2)$ whereas for clinical group $(26.09 \pm 17 \mathrm{~cm} 2)$ and $(27.97 \pm 3.66 \mathrm{~cm} 2)$ respectively. In healthy group all cases showed a thin echogenic line representing the capsule (Fig. 1 and 2). The renal cortex was hypoechoic and finely granular structure. The renal medulla was anechoic and the renal pelvis was seen as an irregular echogenic mass at the hilus of the kidney and these findings was correlated with the findings of Barr F. J., (1990). 


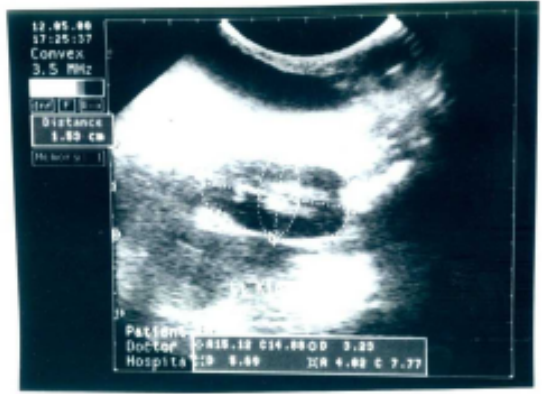

Fig 1. The thin echogenic line marked representing the capsule of Lt. kidney of healthy group

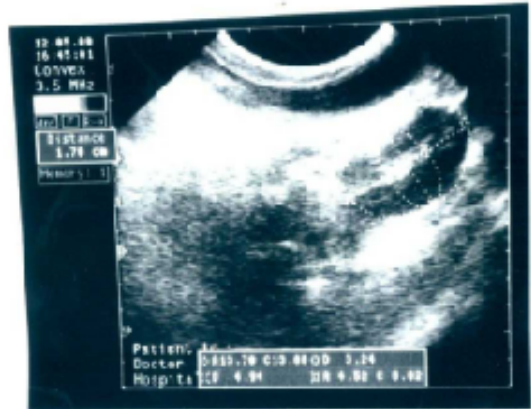

Fig 3. The more echogenic mass in renal pelvis of Lt. kidney of clinical group

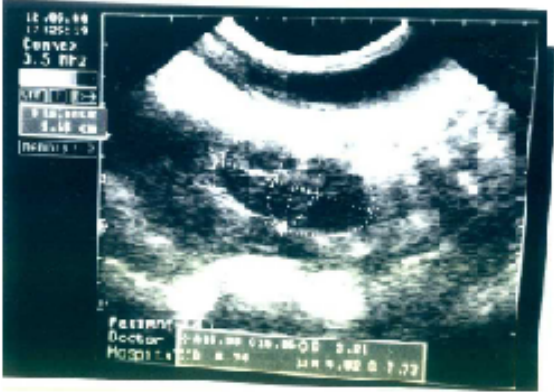

Fig 2. The thin echogenic line representing the capsule of the Rt. kidney of healthy group

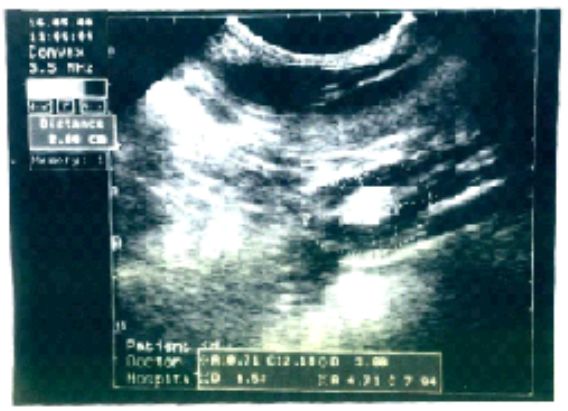

Fig 4. The thickening of capsule of Lt. kidney and reduction of volume of clinical group

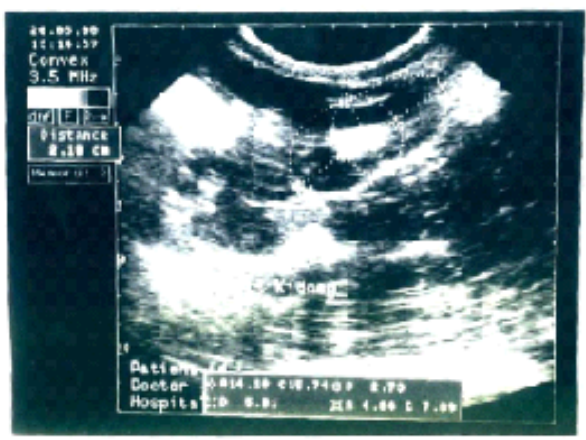

Fig 5. The renal pelvis shows more echogenicity of Lt. kidney of clinical group

The reduction in the both left and right kidney in clinical cases might be due to renal impairment, age factor, renal fibrosis or calcification. Similar findings were reported by Triolo et al. (1995). The Left kidney of one of the clinical cases showed more echogenic mass in renal pelvis as well as in the cortical region that might be due to high fat and fibrous tissue content, (Fig. 3). These findings were in accordance with the findings of Barr (1990). The right kidney of one of the clinical 
cases showed thickening of capsule as well reduction of size, suggestive of renal fibrosis, (Fig. 4). The correlations were in accordance with the findings of Billar et al. (1992). In Fig. 5, showed, slight displacement of kidney and more echogenicity suggestive of fibrosis. Similar findings were noted by Mantis and lamb (2000). The displacement of kidney might be due to spleenomegaly in this case. In the present study amongst the clinical cases the minimum volume of both right and left kidney found to be $19.04 \mathrm{~cm} 2$ and $19.41 \mathrm{~cm} 2$ respectively. From this study it can be inferred that ultrasonography is an useful tool to evaluate the architectural detail of kidney especially the changes in size and shape. Moreover the procedure is noninvasive and much more pragmatic in canine patient.

The present study comprises of 12 dogs, out of which 6 dogs were healthy and 6 were clinical cases. The 6 clinical dogs were evaluated with different parameters for the assessment of renal status and remaining 6 dogs were considered as standard. Ultrasonography was find to be very efficient tool to diagnose the structural abnormality of canine kidney. Different images of canine kidney of clinical cases obtained from sonographic print revealed variation in architectural details comparing with the kidney images of healthy canine group. In the present study after calculation of kidney volume, the left kidney of clinical cases found to be significantly reduced than healthy dogs. Moreover, Mode, frequency and focal range of the transducer influences the appearance of scanning images. To find out the kidney images of obese dog was rather difficult. With this research we can inferred that the change of size and shape of kidney may be due to various chronic renal diseases of canine can be evaluated with the support of Ultrasonography.

\section{REFERENCES}

1. Barr FJ, 1990. Evaluation of ultrasound as a method of assessing renal size in the dog, Journal of Small Animal Practice, 31: 174-179.

2. Biller DS, GA Bradley and BP Partington, 1992. Renal medullary rim sign: Ultrasonographic evidence of renal disease. Veterinary Radiology \& Ultrasound, 33. 286-290.

3. Felkai CS, K Voros, T Vrabely and F Karsai, 1992. Ultrasonography determination of renal volume in dog. Veterinary Radiology \& Ultrasound, 33: 292-296

4. Mantis $P$ and CR Lamb, 2000. Most dogs with medullary rim sign on ultrasonography have no demonstrable renal dysfunction. Veterinary Radiology \& Ultrasound, 41: 164-166

5. Triolo AJ and KG Miles, 1995. Renal imaging techniques in Dogs and Cats, Symposium on diagnostic imaging techniques. Vet. Medicine 91: 959-966.

6. Walter PA, GR Johnson, DA Feeny and TD O'Brien, 1987. Renal ultrasonography in healthy cats. American Journal of Veterinary Research, 52:1357-1365 